\title{
THE ORGANO-IRON COMPOUND IN THE DIGITONIN EXTRACT OF THE ROD OUTERSEGMENT
}

\author{
Shigechika FUJISHITA* \\ Department of Physiology, Osaka University of Liberal Arts \\ and Education, Tennoji-ku, Osaka, Japan
}

KÜHNE $(1878)^{1)}$ paid attention to the fact that iron is present in some animal pigments. He attempted to demonstrate iron in rhodopsin experimentally only to obtain a negative result. The modern advancement in techniques of chemical analysis with organic reagents encouraged the present author to attempt to demonstrate even a small amount of iron possibly existing in a rhodopsin molecule. In 1959 FUKAMI et al. ${ }^{2}$ reported that their experiments offered no evidence for the participation of a metal ion in the structure of rhodopsin. In spite of their conclusion, however, the present author detected a trace of iron in a rhodopsin solution.

The present paper deals with the existence of an organo-iron compound in the digitonin extract of rod outersegment. It refers to two experiments, one dealing with the demonstration of iron in the rhodopsin solution extracted from the outersegment, and the other with methods of testing the existence of iron-containing enzymes in the outersegment.

\section{EXPERIMENT I}

MATERIALS AND TECHNIQUE

1. Materials and flotation technique: Experimental animals employed were frogs (Rana nigromaculata). They were kept in a dark room about three hours. All operations were carried out under dim red light below one lux. Frogs were decapitated and their eye-balls were enucleated. With fine scissors a single cut was made across the equator and each posterior half of the eye-ball was placed in a vessel containing a sucrose solution (specific gravity 1.20). The retinae were removed with a crook tipped glass rod and were crushed between two slide glasses. Then the outersegments of rods were isolated by KIMURA's flotation technique. ${ }^{3)}$

Instead of sodium bicarbonate employed in KIMURA's method, tris-(hydroxymethyl)aminomethane was used to adjust a sucrose solution to $\mathrm{pH} 7$, since a trace of inorganic iron ions was found in a sample of sodium bicarbonate following the staining method with thioglycollic acid. The outersegments collected at the boundary between the two layers of the sucrose solution (specific gravity being 1.15 and 1.12 respectively) were pipetted off. In order to remove sucrose, distilled water (adjusted to $\mathrm{pH} 4$ with

Received for publication March 29, 1962

*藤下成周 
glacial acetic acid) was added to a suspension of the outersegment and then centrifuged at 3000 r. p. m. for $15 \mathrm{~min}$. After the outersegments were precipitated to the bottom of a centrifuge tube, the sucrose contained in the tube was washed out with distilled water three or four times. Then $6 \mathrm{cc}$ of a $1 \%$ digitonin solution was added in the tube and was stirred with a glass rod so as to keep the outersegments suspended in the digitonin solution. After being kept in a dark room at $4^{\circ} \mathrm{C}$ for 20 hours the suspension was centrifuged at $3000 \mathrm{r}$. p. m. for $15 \mathrm{~min}$. and the purple supernatant (rhodopsin solution) was removed into a melting pot.

2. Preliminary tests of water and reagents: During a preliminary test, iron ion was found in the rhodopsin solution. Accordingly the author carried out experiments to confirm the existence of organic iron in rhodopsin molecules. For this purpose, it is required that water, sucrose, tris-methane, glacial acetic acid and digitonin employed in these experiments are free from iron. Therefore these substances were previously tested with thioglycollic acid. In order to obtain an iron free water, distilled water which has been purified by a copper distiller was redistilled by a glass distiller.

3. The thioglycollic acid method for detecting iron: The term 'thioglycollic acid reagent' will be referred to as ' $T$-reagent' in this paper. T-reagent was prepared as follows: First $4 \mathrm{ml}$ of thioglycollic acid were added to $8 \mathrm{ml}$ of aqueous solution of ammonia and mixed. Then the mixture was dissolved in redistilled water, and diluted to $50 \mathrm{ml}$. When several drops of T-reagent are added to a sample, an immediate colouring (violet) indicates the presence of inorganic iron (ferrous and ferric) ions. Ferrous ion reacts with T-reagent directly producing a coloured compound, but ferric ion does not combine with T-reagent primarily. Ferric ion is reduced with thioglycollic acid to ferrous ion, and then combines with thioglycollic acid to form a coloured (violet) compound.

On the other hand, T-reagent can react with cobalt and nickel ions forming a coloured compound (reddish brown and olive green respectively).

\section{RESULTS}

In the first series of 2 experiments, 30 frogs were employed in each case. In each experiment, a rhodopsin solution extracted from the outersegments of rods was removed into a melting pot and was condensed on a waterbath. Accordingly rhodopsin was bleached to a yellow substance. In order to demonstrate the existence of inorganic metal ions, including iron, cobalt and nickel, 6 drops of T-reagent were added to the melting pot. The reaction of metal ions with T-reagent was not observed. This finding seemed to indicate that there was no detectable trace of inorganic metals (such as iron, cobalt and nickel ions) in the rhodopsin solution. After being tested with T-reagent, the sample in a melting pot was dried on a waterbath and heated at $600^{\circ} \mathrm{C}$ in an electric furnace for 10 minutes to be ashed. Then 6 drops of T-reagent were added to the pot. Soon after the addition of the reagent, the pot remained colourless, but in several minutes it gradually changed to pale violet.

In the second series of five experiments, 60 frogs were employed in each case. In each experiment, the rhodopsin solution was dried on a waterbath and then heated at $600^{\circ} \mathrm{C}$ in an electric furnace to be ashed. After being cooled at room temperature, the sample (ashes) was tested with T-reagent. In these 
experiments, the ashes coloured to violet with T-reagent immediately without exception. This finding indicated that all the rhodopsin samples employed contained organic iron ions. But from the above experiments it was not clear that the ions were ferrous or ferric. In order to discriminate ferrous ion from ferric ion, a $1 \%$ dipyridyl solution was used as reagent. (It was reasonably considered that ferrous iron would result in an immediate change of colour to red. On the other hand, when ferric ion and dipyridyl were mixed, no immediate changes in colour were observed, but after two hours ferric ion was changed into ferrous ion by the reduction of dipyridyl, revealing the colour of the solution to be red).

In the last experiment, a dipyridyl solution was used as reagent. A rhodopsin solution prepared from 60 frogs was removed into a melting pot and burnt to ashes in an electric furnace. When 6 drops of dipyridyl reagent were added into the pot, no colour change was observed on the bottom of the pot, but after two hours, the reagent became red. This finding indicates that the iron ion in a bleached rhodopsin solution may be ferric ion.

\section{EXPERIMENT II}

In the first experiment the present author confirmed the existence of an organo-iron compound in an apparently pure rhodopsin solution. However, concerning the presence of iron ion in a rhodopsin molecule a complete knowledge is still lacking. Therefore, in order to determine the origin of the organic iron, it is necessary to carry out experiments for possible etiological substances, such as iron containing enzymes. For this purpose, crude retinae were stained with Nadi-reagent for cytochrome oxidase, and with benzidine for peroxidase. On the other hand, in order to discriminate the above organo-iron compound from hemoglobin, catalase and cytochrome c, a rhodopsin solution was added to a water-alcohol-chloroform mixture.

\section{MATERIALS AND TECHNIQUE}

1. Nadi reaction for cytochrome oxidase: The Nadi reagent was prepared as follows ${ }^{41}$ : Equal parts of an aqueous solution of $0.01 \mathrm{M}$ dimethyl-p-phenylendiamine $\mathrm{HCl}$ and an aqueous solution of $0.01 \mathrm{M} \alpha$-naphtol were combined just before use. The fresh retinae isolated from 30 frogs were incubated in $25 \mathrm{ml}$ of phosphate buffer $(\mathrm{pH} 5.8)$ with $2 \mathrm{ml}$ of Nadi reagent at $25^{\circ} \mathrm{C}$ for 10 minutes. After a 10 minute period, the retinae were crushed between two slide glasses and centrifuged following KIMURA's flotation technique.

2. Benzidine reaction for peroxidase: A reagent was prepared as follows $4: 1$ drop of $\mathrm{H}_{2} \mathrm{O}_{2}$ (commercial) was added to saturated benzidine in a $0.65 \%$ saline solution immediately before use. Retinae were removed into the reagent and let to stand in it for 30-40 seconds. If peroxidase was present in the retinae, added benzidine was oxidized and coloured to deep blue.

3. Catalase, hemoglobin and cytochrome $c$ : Although catalase or cytochrome $\mathrm{c}$ might 
be mingled in a rhodopsin solution, the iron ion contained in this solution could be separated from these enzymes by the following procedure: The rhodopsin solution was added to a $3: 4: 10$ water-99\% alcohol-chloroform mixture, and was stirred vigorously for several minutes, then centrifuged at 1000 r.p.m. for 15 min. Following centrifugation the mixture was separated into three parts (upper layer, lower layer and the boundary between them). The mixture will be referred to as 'WAC-mixture' in this paper.

Preliminary test 1: Catalase solution was prepared from frog erythrocytes. The blood of a frog was diluted by addition of a $0.65 \% \mathrm{NaCl}$ solution and centrifuged at 1000 r.p. m. for $3 \mathrm{~min}$. Then the supernatant was discarded, the erythrocytes being left at the bottom of the test tube. In order to disintegrate the erythrocytes, distilled water was added. After hemolysis, the sample was centrifuged and separated into two parts (supernatant and blood ghost). The supernatant contained hemoglobin and catalase.

The supernatant was added to WAC-mixture, and was stirred vigorously for scveral minutes. Then the mixture was centrifuged, and separated into three parts. Catalase was found in the upper layer and denatured hemoglobin was found at the boundary (PLATE IIA). Each of these three fractions was removed into a melting pot, and was dried on a waterbath. After being dried the sample was burnt to ashes. By a reaction with $\mathrm{T}$-reagent, the ashes originated from the upper layer and the denatured hemoglobin coloured to violet (Fe positive), while the ashes originated from the lower layer remained colourless ( $\mathrm{Fe}$ negative).

Preliminary test 2: A preparation of cytochrome c (commercial) was added to WAC-mixture, and by the procedure described above, three fractions were obtained. The upper layer contained cytochrome $c$, and hence being Fe positive (PlATE IIB). But the present author could not detect a trace of iron ion with $\mathrm{T}$-reagent in the lower and in the denatured protein which was collected at the boundary between the two layers.

RESULTS

1. Nadi reaction for cytochrome oxidase. The isolated outersegments remained purple, while the other parts of retinae were stained blue with the Nadi reagent (PLATE I). This finding suggested that there was no cytochrome oxidase in the outersegment of rod.

2. Benzidine reaction for peroxidase. The retinae did not change to deep blue with the benzidine reagent. This finding suggested that there was no peroxidase in frog retina.

3. Detection for catalase, hemoglobin and cytochrome $c$. A rhodopsin solution was added to WAC-mixture and stirred vigorously. By centrifugation the mixture was separated into three parts. The lower layer contained yellow substance and iron ion, but no iron was found in the upper layer and in the denatured protein which was collected at the boundary between the two layers (Plate IIC).

As described above (preliminary test 1 and 2), catalase and cytochrome $c$ were found in the upper layer and denatured hemoglobin was collected at the boundary between the two layers (PLATE IIA and IIB). But the iron ion in the rhodopsin solution was shifted to the lower layer (the chloroform layer). Thus, 
it does not seem likely that the origin of the iron ion in the rhodopsin solution was hemoglobin, catalase or cytochrome c.

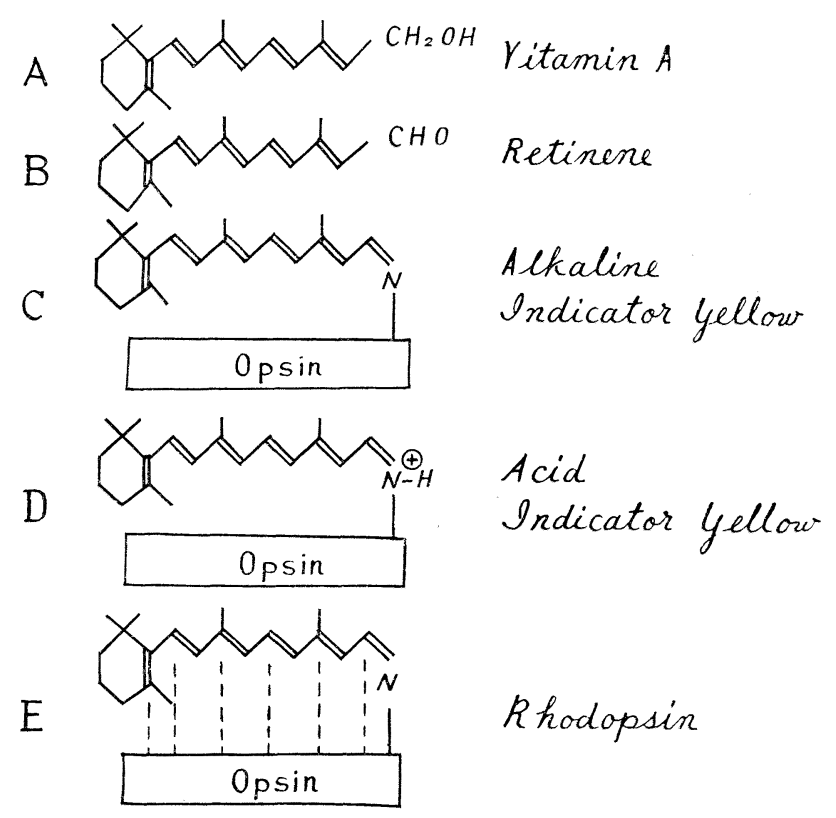

FIG, 1.

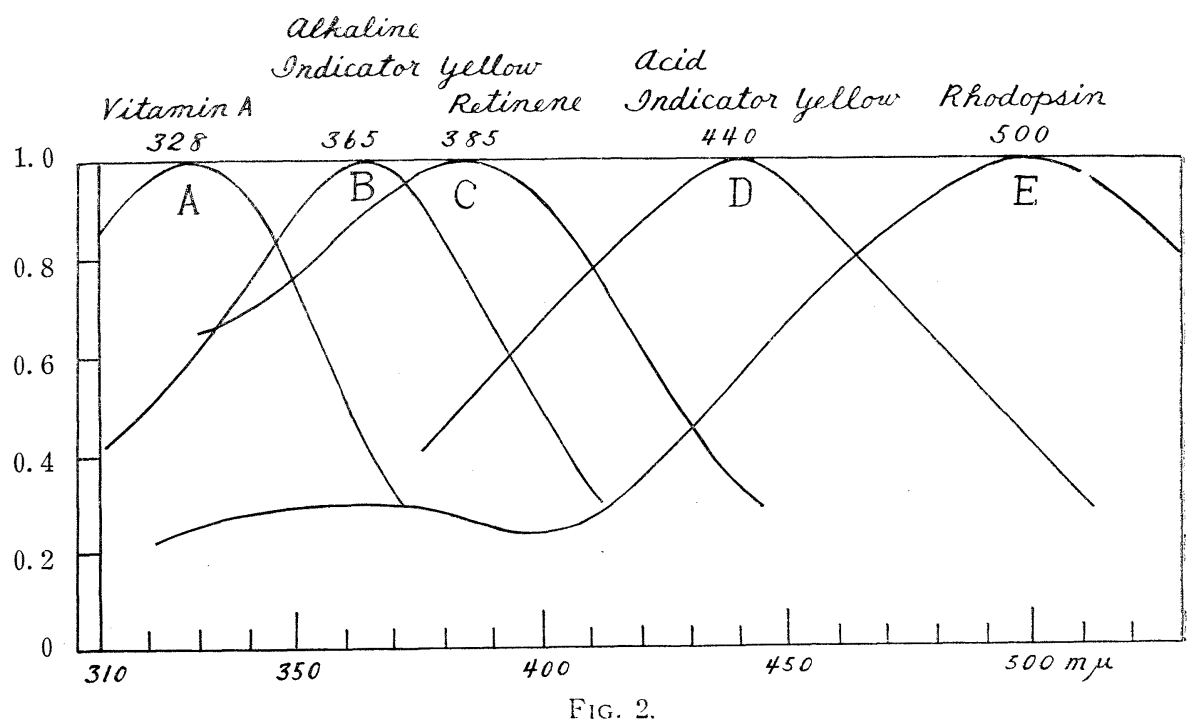

Fig. 2. 


\section{DISCUSSION}

Vitamin A, retinene, alkaline indicator yellow and acid indicator yellow have the respective formulae of $\mathrm{A}, \mathrm{B}, \mathrm{C}$ and $\mathrm{D}$ (FIG. 1). The formula of rhodopsin has hitherto been reported by several authors, but concerning the interaction of opsin with retinene, much remains yet to be studied. From a synthetic point of view WALD and BROWN ${ }^{5}$ suggested that rhodopsin is 'retinene-S-opsin', while on the analytic evidence MORTON and his colleagues ${ }^{6)}$ affirmed that it is 'retinene-N-opsin'. Both rhodopsin and indicator yellow have retinene as a chromophore group and have opsin as a protein moiety, but rhodopsin differs from indicator yellow in terms of $\lambda_{\max }$ in the absorption spectrum (FIG. 2). The difference may be due to the difference following interactions of opsin with retinene. Namely, in rhodopsin $\pi$-electrons may participate in the interaction of opsin with retinene, but in indicator yellow $\pi$-electrons remain free.

The chromophore of rhodopsin is bound to opsin at a number of points. It would seem likely that one bond ties through a nitrogen atom, but other bonds have been left unknown. The colour (purple) of a rhodopsin molecule may be attributed to unknown bonds between retinene and opsin. In this regard, the present author considered that a metal ion involved in these unknown bonds may play an important role in the appearance of the colour. As reported in experiment I, it was confirmed that the samples of rhodopsin solution were constantly associated with iron, while KüHNE could not demonstrate iron in the frog retina, nor could FUKAMI et al. in the cattle retina. It would seem unlikely that inorganic iron ions were adsorbed by rhodopsin molecules during the procedure of preparation, since no iron ion was detected in the sucrose solution with T-reagent. However, the existence of iron ion in a rhodopsin molecule can not be inferred merely from the findings described in experiment $I$, because it is possible that during the preparation, hemoglobin or one of the iron-containing enzymes may mingle into these solutions. In order to demonstrate the existence of cytochrome oxidase, crude retinae were stained with Nadi reagent and centrifuged by KIMURA's flotation technique. The separated outersegments remained purple. Peroxidase was not demonstrated by the benzidine reaction in a crude frog retina. For the purpose of separating the organo-iron compound from hemoglobin, catalase and cytochrome c, samples were added to WAC-mixture and stirred vigorously. After centrifugation, cytochrome $\mathrm{c}$ and catalase were found in the upper layer, and hemoglobin was found at the boundary between the upper and the lower layers, while the organo-iron compound was dissolved in the lower layer. This finding indicates that hemoglobin, cytochrome $c$ and catalase can not be the origin of the organic iron in a rhodopsin solution. Therefore, it is reasonable to consider that a rhodopsin molecule involves at least one iron ion as a component. 
As described above, the absorption spectrum of rhodopsin has never been completely analysed from a view point of its molecular structure. Therefore, the present author attempted to clarify a contribution of chemical structure of rhodopsin to the absorption spectrum. If, as is likely, any iron ion is present between opsin and retinene, linkage may be considered connecting active groups of opsin (such as $-\mathrm{SH},-\mathrm{NH}_{2},-\mathrm{COOH}$ or $-\mathrm{OH}$ ) with iron, simultaneously connecting the electrons of the iron with the cloud of $\pi$-electrons of retinene. The $\lambda_{\text {nax }}$ at $500 \mathrm{~m} \mu$ of the absorption spectrum of rhodopsin may be attributed to the passage of a $\pi$-electron to the iron.

\section{SUMMARY}

1. Iron ion was found in an apparently pure rhodopsin solution, which was extracted from the outersegments of rods of frog retinae.

2. No demonstration was made of the existence of cytochrome oxidase and peroxidase in the outersegments of rods of frog retinae with Nadi and benzidine reagents respectively.

3. The organo-iron compound contained in rhodopsin solution was differentiated from heme-proteins such as hemoglobin, cytochrome $\mathrm{c}$ and catalase, by a separation technique with a water-alcohol-chloroform mixture.

4. A rhodopsin molecule seemed to contain at least one iron ion.

5. Discussion was made of a possibility that the $\lambda_{\max }$ at $500 \mathrm{~m} \mu$ of the absorption spectrum of rhodopsin was attributable to iron ion.

The author wishes to thank Prof. Y. Hosoya and Prof. E. Kimura for their helpful suggestions and criticism during in finishing the present study.

\section{REFERENCES}

1) KüHne, W. Enthält der Sehpurpur Eisen? in : Unters. Physiol. Inst. Univ. Heidelberg. 1: 438-440. 1878 .

2) Fukami, I., Vallee, B. L. and Wald, G. Does rhodopsin contain a trace metal? Nature 183: 28-30, 1959.

3) Kimura, E. A new method of separating the outersegments of rods from retinal tissues. Jap. J. Physiol. 3 : 250-253, 1952.

4a, 4b) Pearse, A. G. E. Histochemistry, 2. Ed., pp. 510-514, 517-520. London. : J. \& A. Churchill, 1960.

5) WALD, G. and Brown, P.K. The role of sulfhydryl groups in the bleaching and synthesis of rhodopsin. J. gen. Physiol. 35 : 797-821, 1952.

6) Collins, F. D. and Morton, R. A. Studies in rhodopsin. 3 : Rhodopsin and transient orange. Biochem. J. $47 ; 18-24,1950$. 


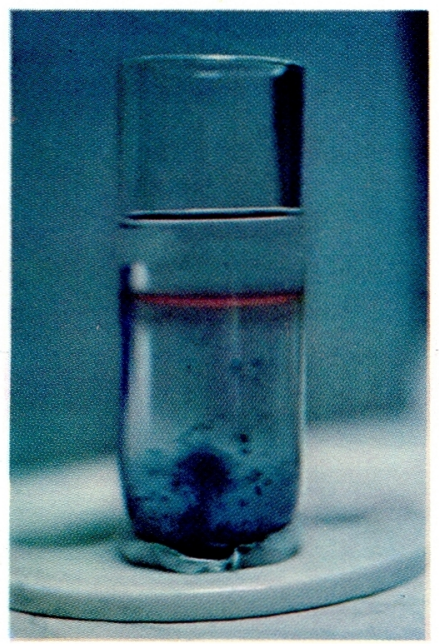

\section{PLATE I.}

After being incubated in a mixture of phosphate buffer and Nadi reagent at $25^{\circ} \mathrm{C}$ for $10 \mathrm{mi}$ nutes, the retinae of frogs were crushed and centrifuged by Kimura's flotation technique. The outersegments collected at the boundary between two sucrose layers (specific gravity being 1.12 and 1.15 respectively) remained purple. This fact indicates the absence of cytochrome oxidase in the outersegments.

\section{Catalase}

AND

Cytochrome c Rhodopsin

\section{Hemoglobin}

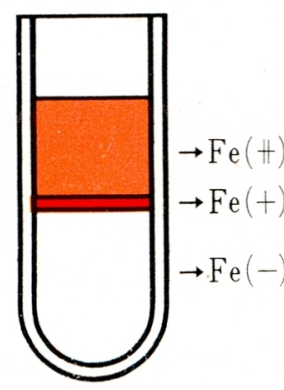

A

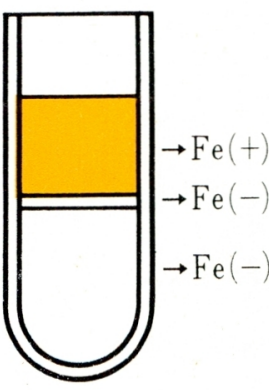

B

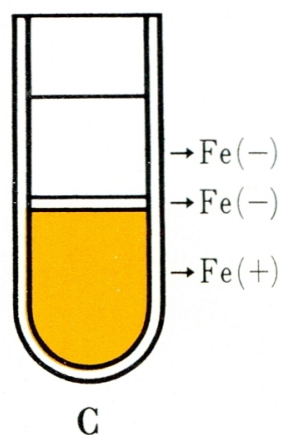

C

PLATE II.

A 3:4:10 water-alcohol-chloroform mixture was added to a sample of hemoglobin, catalase, cytochrome c or rhodopsin, and was centrifuged at $1000 \mathrm{r} . \mathrm{p} . \mathrm{m}$. for $15 \mathrm{~min}$. Following centrifugation the mixture was separated into three parts.

A : When a sample of disintegrated erythrocytes containing catalase and hemoglobin was treated with the mixture, catalase and hemoglobin were found in the upper layer and at the boundary between the two layers respectively.

$\mathrm{B}$ : Cytochrome c was found in the upper layer.

C : Rhodopsin (bleached) was found in the lower layer. 